\title{
Review Article \\ Optogenetics: Novel Tools for Controlling Mammalian Cell Functions with Light
}

\author{
Toshihiro Kushibiki, Shinpei Okawa, Takeshi Hirasawa, and Miya Ishihara \\ Department of Medical Engineering, National Defense Medical College, 3-2 Namiki, Tokorozawa, Saitama 359-8513, Japan \\ Correspondence should be addressed to Toshihiro Kushibiki; toshi@ndmc.ac.jp
}

Received 13 December 2013; Revised 7 February 2014; Accepted 9 February 2014; Published 28 April 2014

Academic Editor: Rudolf Steiner

Copyright ( 2014 Toshihiro Kushibiki et al. This is an open access article distributed under the Creative Commons Attribution License, which permits unrestricted use, distribution, and reproduction in any medium, provided the original work is properly cited.

In optogenetics, targeted illumination is used to control the functions of cells expressing exogenous light-activated proteins. Adoption of the optogenetic methods has expanded rapidly in recent years. In this review, we describe the photosensitive channel proteins involved in these methods, describe techniques for their targeting to neurons and other cell types both within and outside the nervous system, and discuss their applications in the field of neuroscience and beyond. We focus especially on the channelrhodopsin protein ChR2, the photosensitive protein most commonly employed in optogenetics. ChR2 has been used by many groups to control neuronal activity, both in vitro and in vivo, on short time scales and with exquisite anatomical precision. In addition, we describe more recently developed tools such as opsin/G protein-coupled receptor chimeric molecules and a light-activated transgene system. In addition, we discuss the potential significance of optogenetics in the development of clinical therapeutics. Although less than a decade old, optogenetics is already responsible for enormous progress in disparate fields, and its future is unquestionably bright.

\section{Introduction}

Optogenetics comprises a growing family of related techniques in which genetically modified cells are stimulated by light in order to influence cellular behaviors. For target cells to be influenced by light, they must be engineered to express exogenous photosensitive proteins that alter membrane potential, or other cellular properties, in response to illumination [1-5]. The effects of membrane depolarization (or, less commonly, hyperpolarization) can then be monitored at the level of individual cells, groups of interacting cells (e.g., tissues or neural circuits), or the whole organism. Therefore, optogenetics encompasses several components: discovery and optimization of photosensitive proteins, techniques for targeting the genes encoding these proteins to specific cell types, and technologies for targeted illumination in vivo. In addition, optogenetics is a suitable method for observing the electrophysiological, functional, and behavioral changes resulting from photostimulation [6-10].

The field of optogenetics is quite young. Several of the light-sensitive proteins used in optogenetic experiments, such as channelrhodopsin [11, 12] and halorhodopsin [1315], were first identified decades ago. However, it was not until 2005 that they were first used to control neuronal activity [16]. The term "optogenetics" was itself coined around 2006 by Deisseroth, whose laboratory pioneered many of the genetic and optical techniques that facilitate the use of illumination to investigate neural dynamics [17]. The earliest studies were performed in vitro in cell culture, but by 2007 , the advent of fiber-optic neural interfaces permitted the use of bacterial opsins to influence behavior in intact, freely behaving mammals $[18,19]$. Since that time, the optogenetic applications have expanded rapidly to encompass studies of neural circuits, brain diseases, and nonneuronal systems such as stem cells, cardiac tissue, and skeletal muscle. Accordingly, based on its growing importance as a paradigm in many fields of biomedical research, optogenetics was selected as the "Method of the Year" in 2010 by Nature Methods [20].

In this review, we summarize the recent literatures describing the use of optogenetics to develop novel technologies for control of cellular functions. Because most of the literature published to date describes the use of photoactive 

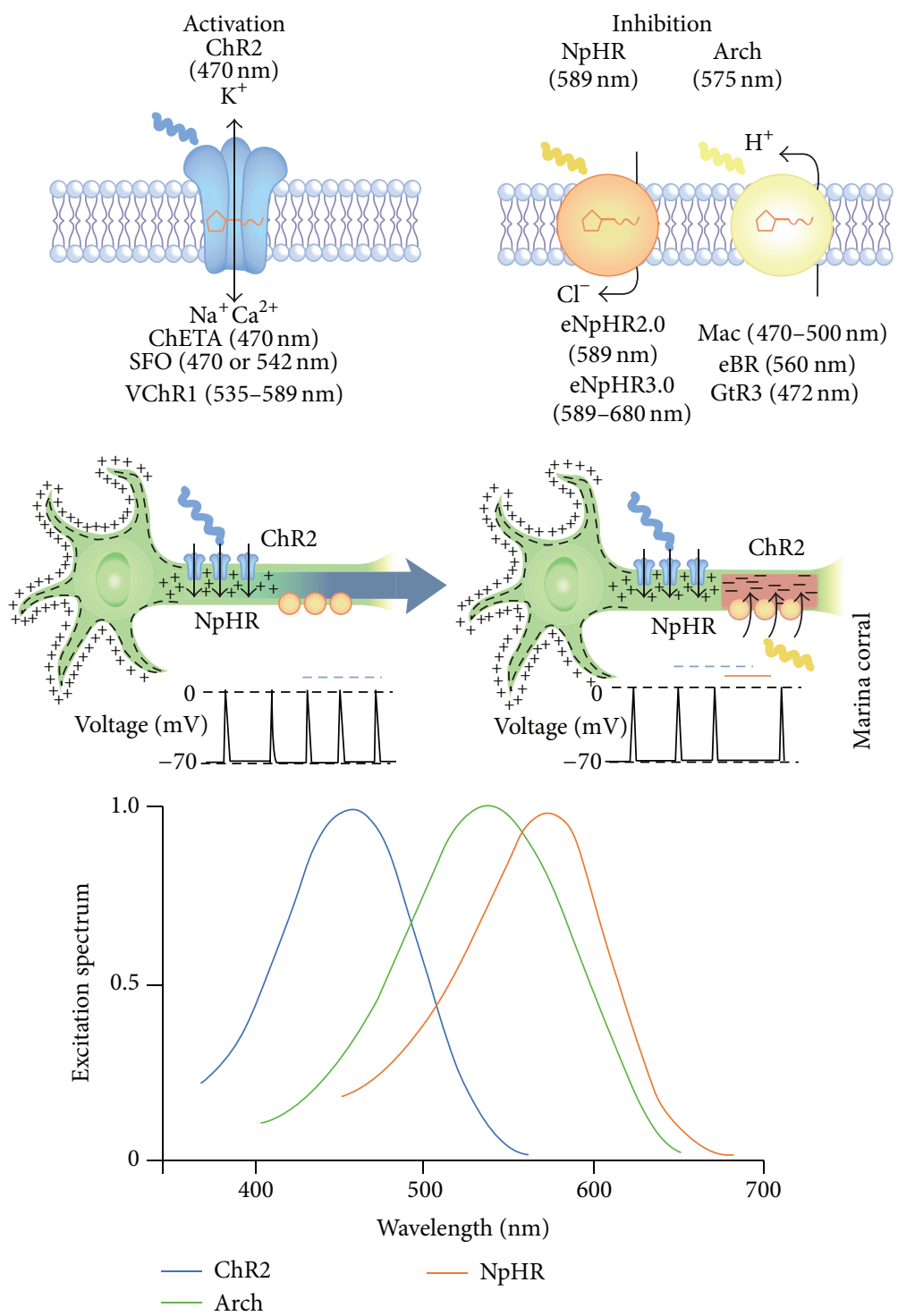

FIGURE 1: Optogenetic tools for modulating membrane voltage potential. Adapted with permission from Macmillan Publishers Ltd. (Nature Publishing Group): Nature Methods [7], copyright (2011).

proteins, such as microbial opsins and plant light sensors, we provide an overview of these representative photoactive proteins. In addition, we summarize the clinical significance of optogenetics.

\section{Photoactive Proteins}

2.1. Channelrhodopsin and Halorhodopsin. In general, the optogenetics manipulations involve altering the membrane potential of electrically excitable cells by illuminating them with controlled bursts of light. Membrane depolarization of neurons induces transient electrical signals (spikes or action potentials) that ultimately convey information between neurons within the same circuit. For neurons to be artificially depolarized by illumination, they are engineered to express proteins that alter the ion permeability of membranes in response to light. The first light-sensitive protein used as a "switch" to control neuronal activity was the Chlamydomonas protein channelrhodopsin-2 (ChR2), a cation selective channel that permits entry of $\mathrm{Na}^{+}$and $\mathrm{Ca}^{2+}$ ions in response to blue light (470 nm wavelength) [21-31] (Figure 1). ChR2, an algal phototaxis receptor that uses light to depolarize the plasma membrane [32], acts as a light-gated cation channel when expressed in animal cells [12]. ChR2 is expressed mainly under low-light conditions, suggesting that the protein is involved in photoreception in dark-adapted Chlamydomonas cells. In contrast to the most cation-selective ion channels, ChR2 contains the seven transmembrane alpha helices characteristic of G protein-coupled receptors (GPCRs). Nagel et al. demonstrated that ChR2 could be used to depolarize the cells of various sizes simply by illuminating cells with the appropriate wavelength [12]. When ChR2 was expressed in a neuron and the cell was exposed to blue light, the channel immediately depolarized the neuron, triggering a spike. 
Beginning with cultured neurons and proceeding to more elaborate in vitro systems, this property of ChR2 has been exploited to precisely control neuronal activity. In an initial study, the Deisseroth group [16] used ChR2 to reliably control neuronal spiking on millisecond timescales to control both excitatory and inhibitory synaptic transmissions. Because illumination can be controlled at very high spatial and temporal resolution, this approach permits experimental manipulation of neural processing with great precision, even at the level of single spikes and synaptic events. Millisecond- to second-scale control of neuronal function was also achieved by $\mathrm{Li}$ et al. [33], who developed systems for light activation of neurons using vertebrate rat rhodopsin 4 (RO4) and green algae ChR2. Their use of two light-activated proteins permitted precise and reversible antagonistic control of neuronal function, as demonstrated in both cultured neurons and intact spinal cords. Antagonistic control was also achieved by using alternative light-activated proteins with different wavelength sensitivities and ion permeabilities. For example, the chloride pump halorhodopsin (NpHR) hyperpolarizes neurons in response to yellow light ( $590 \mathrm{~nm}$ wavelength), in contrast to $\mathrm{ChR}$, which depolarizes expressing cells under illumination with blue light (Figure 1).

In addition to cultured neurons, ChR2 has been exploited in the context of cultured brain slices also, allowing investigations in larger and more complex arrangements of cells. Zhang and Oertner [34] combined millisecond-scale activation of ChR2 with two-photon calcium imaging of slice cultures of rat hippocampus. In that study, the influx of calcium resulting from light-activated action potentials was greater than those resulting from somatic current injection; consequently, the authors were able to achieve highly reproducible synaptic transmission. Furthermore, correlation of light stimulation with postsynaptic depolarization in the slices induced long-term potentiation. Such in vitro systems will permit investigations of synaptic plasticity using the tools of both genetics and pharmacology.

Slice cultures had been used to address the challenges related to single-cell targeting of optogenetic activation with temporal precision comparable to spike timing. In hippocampal slices from mouse, Andrasfalvy et al. [35] used optogenetic methods to achieve submillisecond control of single neurons. The use of temporally focused laser illumination in that study allowed simultaneous excitation of large numbers of channels on individual neurons, resulting in depolarizations that were both large in magnitude (up to $\sim 15 \mathrm{mV}$ ) and rapid (less than $1 \mathrm{~ms}$ ). The high spatiotemporal resolution of their technique enabled selective illumination (and therefore selective activation) of subcellular features, including presynaptic terminals and dendrites, at considerable depth within the slice.

Although brain slices are more physiologically relevant than cultured individual neurons, they are still not intact brains. Illumination methods optimized for in vivo contexts (such as optical fibers), in combination with transgenic technologies, have enabled ChR2-mediated optogenetics to leap out of the culture dish into live organisms. For example, in the nematode C. elegans, ChR2 expressed in excitable cells has been used to activate specific neurons and muscles, and thereby elicit specific behaviors in living and intact animals [36]. However, the greatest strengths of optogenetics have been revealed by extensive recent studies in rodents. Early studies employed transgenic animals expressing lightactivated proteins throughout the brain, as in a study by Arenkiel et al. [37] using mice expressing a ChR2-YFP fusion protein in the CNS. Those authors were able to exploit in vivo activation of neurons to map neural circuits involved in olfaction; their data suggested that olfactory processing in mouse depends on mitral cell convergence onto olfactory cortex and subsequent integration by cortical cells. This work sets an early precedent for the value of optogenetics methods for influencing neuronal activity in the brains of intact animals and of using such experimental manipulations to probe the complex functional connections between neurons in vivo.

Even greater experimental power has been achieved by targeting the expression of ChR2 to specific subsets of neurons within the brain. Such targeting is often achieved by restricting channel expression both anatomically, by precise delivery of ChR2-encoding viral vectors to specific brain regions, or genetically, by placing $\mathrm{ChR} 2$ under the control of a promoter specifically activated in the cell type of interest. Genetic targeting can itself be achieved by multiple means, for example, by transgenesis or the use of viruses encoding channels driven by cell type-specific promoters. Furthermore, anatomical and genetic targeting methods are often used together. Examples of several targeting approaches and their applications are described below.

In an example of strictly anatomical targeting, Huber et al. [38] performed in utero electroporation of ChR2-GFP into neocortical pyramidal neurons of embryonic mice. As adults, the electroporated mice expressed the channel in a small fraction of layer $2 / 3$ neurons, primarily in the barrel cortex. These animals could be conditioned to detect brief sequences of light pulses (resulting in corresponding brief trains of action potentials), demonstrating that very short periods of cortical activity in a sparse subset of cortical neurons can drive both decision making and learning. In a case of strictly genetic targeting, Wang et al. [39] generated transgenic mouse lines that express ChR2 in various subsets of neurons in different regions of the brain and used their system to map the synaptic circuits of cortical neurons in living animals.

Several studies have targeted delivery of ChR2 both genetically and anatomically in order to restrict expression of the channel to specific brain regions and/or cell types of interest. Tsai et al. [40] stereotactically injected a Cre (site-specific DNA recombinase)-inducible adeno-associated virus (AAV) encoding Chr2-EYFP into the ventral tegmental area (VTA) of tyrosine hydroxylase-internal ribosomal entry site (IRES) - Cre transgenic mice, resulting in expression of the light-activated protein specifically in the dopaminergic (DA) neurons of VTA. By selectively stimulating these cells in freely behaving animals, the authors were able to show that phasic activation of DA neurons in VTA resulted in behavioral conditioning that could not be achieved by longer, lower-frequency action potentials. Similarly, Kravitz et al. [41] injected AAV encoding Cre-activatable ChR2-EYFP 
into dorsomedial striatum of transgenic mice expressing Cre in medium spiny neurons (MSNs), thereby restricting expression of the channel to direct- or indirect-pathway MSNs. Using this system, the authors showed that directpathway activation via fiber-optic illumination of ChR2 rescued disease-associated symptoms in a model of Parkinson's disease (PD). Their findings clearly demonstrated that the basal ganglia circuitry played a crucial role in regulation of parkinsonian motor behaviors and suggested that modulation of direct-pathway circuitry might be used therapeutically to treat the motor deficits associated with PD. In yet another example of combined anatomical and genetic targeting, Adamantidis et al. [18] introduced a lentivirus encoding ChR2-mCherry under the control of the hypocretin (Hcrt) promoter via stereotactic injection into the lateral hypothalamus of mice, resulting in channel expression in a subset of cells within this brain region. Specific patterns of stimulation of Hcrt neurons increased the likelihood of arousal from sleep, demonstrating that these cells played an active role in determining wakefulness. These results provided critical insights into the neural basis of sleep regulation.

It may be possible to achieve even higher spatial resolution, at the level of particular structures within specific cell types, using light-activated proteins that are targeted to subcellular compartments within neurons. (As noted above, this level of resolution can be achieved in brain slices using temporally focused lasers, but this mode of illumination is not optimal for use in the intact brain.) For example, Lewis et al. [42] showed that ChR2 fused to the myosin-binding domain of Melanophilin is localized to the somatodendritic compartment. In conjunction with the anatomical and genetic targeting methods described above, fusion proteins of this sort could be used to enable specific photostimulation of dendrites of particular cell types, dramatically increasing the potential power of circuit-level analyses.

The abovementioned reports suggest that ChR2 had widely been used to modulate the membrane potential in neurons, but recently, several groups also explored the possibility of ChR2 mediating optogenetic application in the brain cells other than the neurons. For example, in order to mimic the $\mathrm{pH}$-induced calcium responses involved in regulation of breathing, Gourine et al. [43] used light to stimulate ChR2-expressing astrocytes. This stimulation resulted in activation of chemoreceptor neurons and influenced respiratory responses in live animals, indicating that glial cells are critically involved in the breathing reflex. Furthermore, ChR2 is increasingly being applied outside the central nervous system altogether. In particular, optogenetics methods have been used to control cardiomyocytes and other electrically active cells in the heart. Bruegmann et al. [44] expressed ChR2 in heart muscle, allowing them to stimulate cardiac tissue with spatial and temporal precision; their findings revealed the effects of prolonged depolarization on rhythm generation and calcium homeostasis in the heart. Skeletal muscle has also been investigated with the tools of optogenetics: Asano et al. [45] used light to induce action potentials in ChR2-expressing myotubes, ultimately resulting in controllable contractions of these cells. Because skeletal muscle transduces force effectively, such an approach could be useful in biological microdevices in which contractile patterns are regulated by localized illumination. In addition, ChR2 has also been introduced into embryonic stem cells (ESCs), allowing reliable tracking of these cells and their progeny, as well as spatial and temporal control of the electrical activity of neurons (and other cell types) derived from ESCs [46, 47]. These approaches should allow precise optical regulation of the differentiation of ESCs both in vivo and in vitro and facilitate studies of the ways that transplanted ESCs contribute to the tissues and networks in which they ultimately reside.

\subsection{Opsin/G Protein-Coupled Receptor (GPCR) Chimeras} and Other Opsins. As noted above, microbial opsins share structural similarities with GPCRs. Airan et al. exploited these similarities to construct opsin-GPCR chimeras, termed "optoXRs," whose signaling activities are responsive to light [48]. In conjunction with the light illumination technologies also used for ChR2-mediated optogenetics, optoXRs were used to precisely control intracellular signaling events in live cells and intact organisms. In their initial study, Airan et al. had developed two optoXRs that activate distinct signaling modules upon light illumination (Figure 2(a)). In the nucleus accumbens of living mice, these two optoXRs exhibited antagonistic effects on neuronal activity. Furthermore, when photostimulation was administered with specific timing, the influence of the optoXRs on intracellular signaling could modulate conditioning in freely moving animals. Thus, the use of optoXRs complements the use of ChR2 mediated for optogenetic control of behavior in mammals such as mice (Figure 2(b)).

In an analogous experiment, Gutierrez et al. [49] developed an optogenetic system in which vertebrate rhodopsin, a $G_{i / o}$ protein-coupled receptor, was expressed in cerebellar Purkinje cells (PCs) of mice. In vivo activation of rhodopsin by light illumination specifically expressed in PCs reduced simple spike firing that was comparable with the reduction in firing observed for the activation of cerebellar $G_{i / o}$-coupled $\mathrm{GABA}_{\mathrm{B}}$ receptors. In particular, the light illumination of the cerebellar vermis in freely moving mice changed the motor behavior. Thus, spike modulation via $\mathrm{G}_{\mathrm{i} / \mathrm{o}}$-mediated signaling in cerebellar PCs affected motor coordination and showed a new promising approach for studying the physiological function of $G$ protein-coupled receptor-mediated signaling in a cell type-specific manner. Karunarathne et al. [50] also exploited nonrhodopsin opsins in an optogenetic paradigm to target several classes of $G$ protein-mediated intracellular signaling in precisely delineated areas of individual cells. Using this system, they were able to modulate behavior of polarized cells and early differentiation of neurons via controlled illumination. A similar strategy could be readily applied to investigate the roles of $G$ proteins in other phenomena, such as the migration and homing of immune cells or the contractions of cardiac tissue.

2.3. Light-Switchable Transgene System. The light sensors used in the systems described above are membrane proteins that localize to the cell surface. They either alter membrane potential or activate intracellular signaling cascades in 


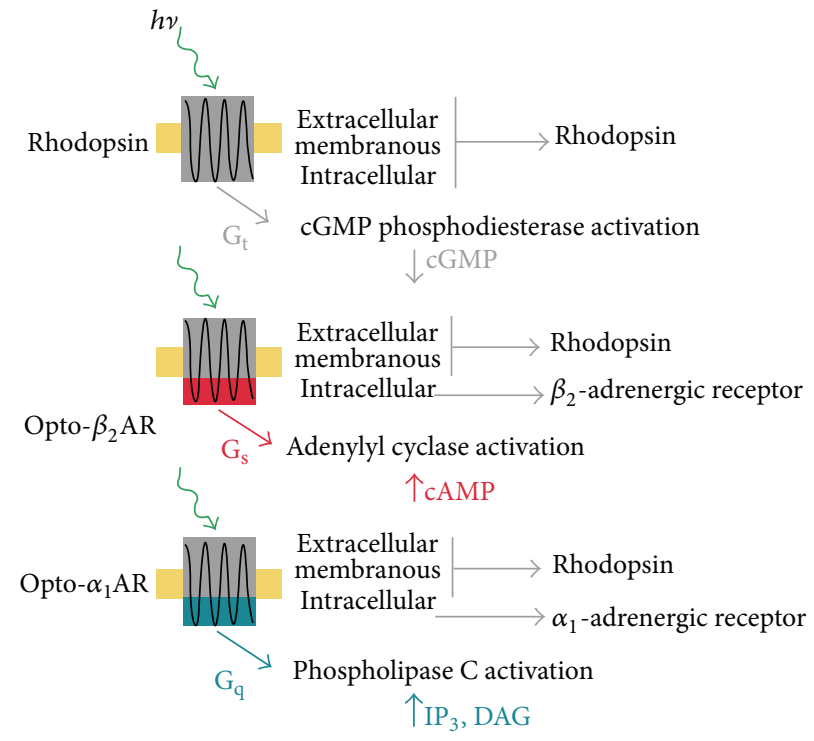

(a)

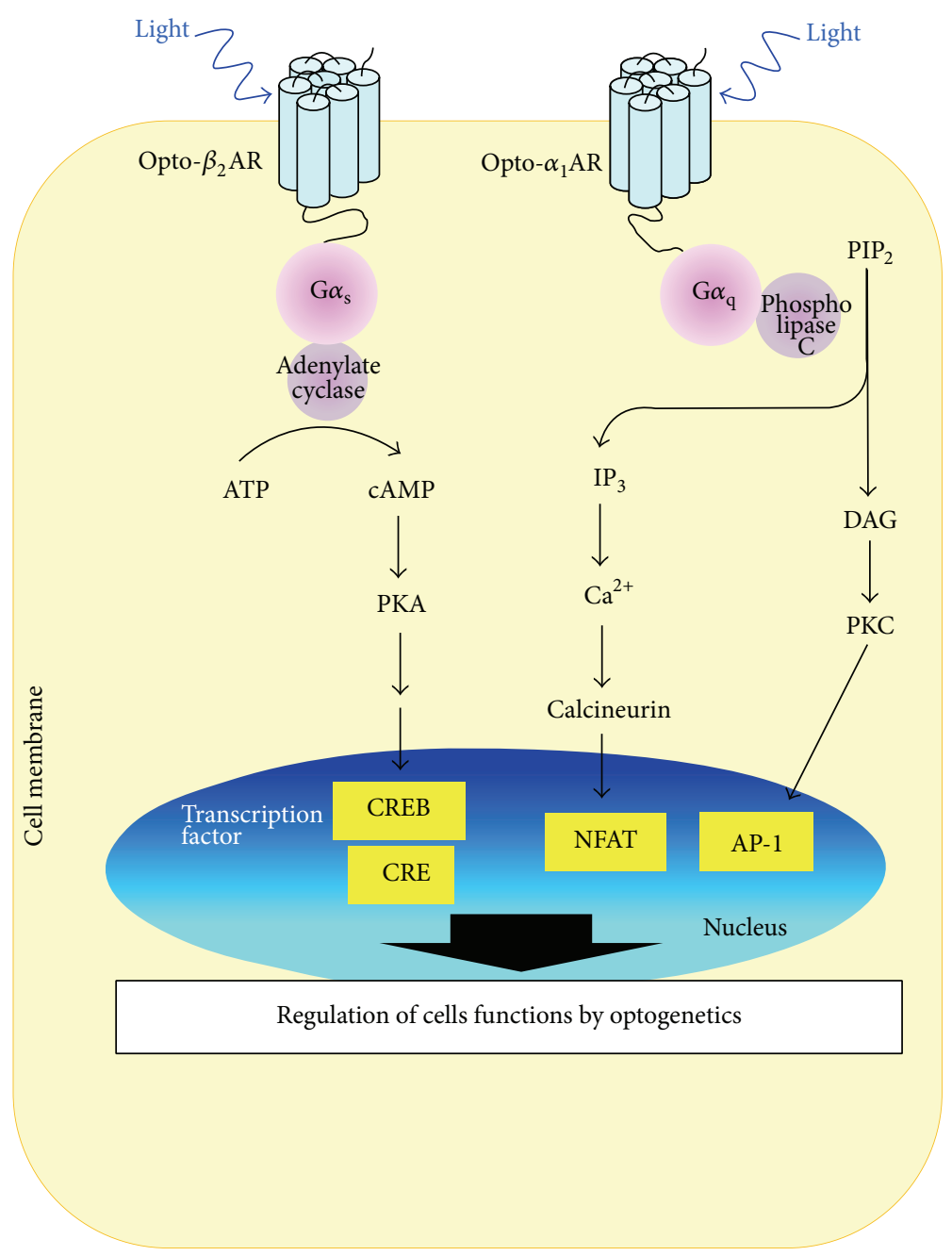

(b)

FIGURE 2: OptoXR: optogenetic control of intracellular signal transduction. (a) OptoXR design. Adapted with permission from Macmillan Publishers Ltd. (Nature Publishing Group): Nature [48], copyright (2009). (b) Possible intracellular signaling after the light illumination of OptoXR. 


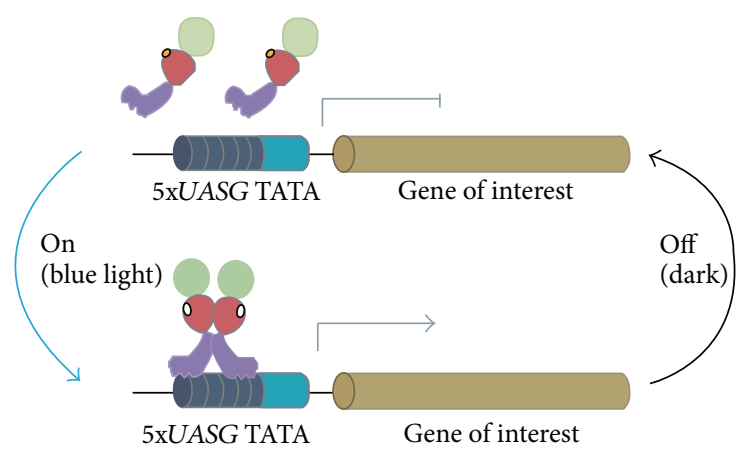

(a)
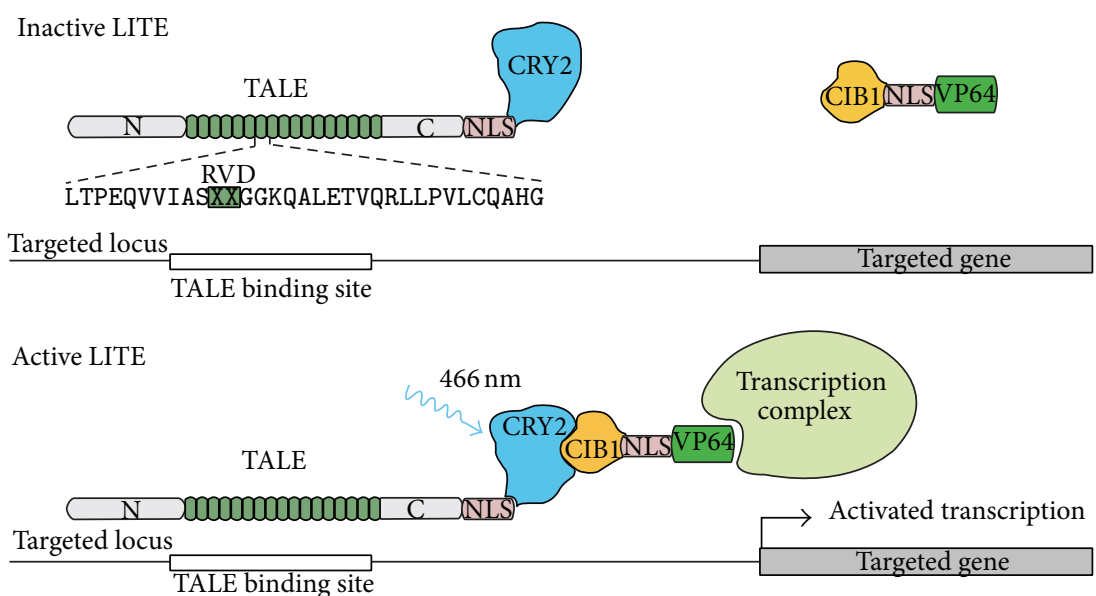

(b)

FIgURE 3: (a) Schematic representation of the LightOn system. After light activation, GAVP homodimerizes, interacts with UASG elements (5xUASG), and initiates expression of the gene of interest. Adapted with permission from Macmillan Publishers Ltd. (Nature Publishing Group): Nature Methods [51], copyright (2012). (b) Schematic of the LITE system. Light stimulation induces dimerization of CRY2 and CIB1, recruiting the effector to the target promoter. Adapted with permission from Macmillan Publishers Ltd. (Nature Publishing Group): Nature [63], copyright (2013).

response to light. Although light activation of these proteins certainly influences gene expression, the specificity of this regulation is quite low and moreover is limited to the downstream targets of the signaling pathways with which the light sensors communicate. In order to overcome these challenges and achieve highly specific gene activation by photostimulation, Wang et al. designed a synthetic lightswitchable transactivator that binds to target promoters in response to blue light [51-53]. The core of the transactivator consists of the well-characterized Gal4 DNA-binding domain fused to VVD, which contains a light-oxygen-voltage (LOV) domain that dimerizes upon blue-light illumination. When this core was fused to be a suitable transactivation domain (e.g., from p65), the fusion protein homodimerized in response to light, bound to the Gal upstream activating sequence, and activated transcription of a target gene or genes (Figure 3(a)). This transgene system, "LightOn," allows spatially and temporally precise manipulation of gene expression levels with far greater specificity than could be achieved by stimulation of membrane proteins that act via intracellular signaling cascades.
The LOV domain was also exploited by $\mathrm{Wu}$ et al. [54] to generate photoactivatable derivatives of Racl, a critical regulator of the actin cytoskeleton. In this system, in unilluminated cells, the LOV domain blocks Racl's interactions with its partner proteins; irradiation causes a linker region connecting Racl and the LOV domain to unwind, liberating Racl to engage in downstream signaling. Using photoactivatable Racl (PA-Rac1), Wu et al. could use light to induce cell protrusions and ruffling with high spatial precision, stimulate cell motility, and influence the direction of movement.

The LOV domain is not the only protein module that might be exploited in strategies such as those described above. Known photoreceptors are distinguished into six classes according to their chromophores and photochemistry: LOV sensors, xanthopsins, phytochromes, blue-light sensors that use flavin adenine dinucleotide, cryptochromes, and rhodopsins [55-57]. Several reviews offer an in-depth discussion of natural LOV and phytochrome photoreceptors $[58,59]$.

Since we have reported that cryptochrome 2, one of the blue light photoreceptors and circadian clock proteins, is 
the key factor controlling cell differentiation after blue light illumination [60-62], cryptochrome 2 is one of the applicable proteins for optogenetics of light-switchable transgene system. Konermann et al. reported the development of lightinducible transcriptional effectors (LITEs), an optogenetic two-hybrid system integrating the customizable TALE DNAbinding domain with the light-sensitive cryptochrome 2 protein and its interacting partner CIB1 (Figure 3(b)) [63]. LITEs do not require additional exogenous chemical cofactors, are easily customized to target many endogenous genomic loci, and can be activated within minutes with reversibility. They have applied that system in primary mouse neurons, as well as in the brain of freely behaving mice in vivo to mediate reversible modulation of mammalian endogenous gene expression as well as targeted epigenetic chromatin modifications. They conclude that the LITE system established a novel mode of optogenetic control of endogenous cellular processes and enables direct testing of the causal roles of genetic and epigenetic regulation in normal biological processes and disease states [63].

2.4. Photoactivated Adenylyl Cyclase (PAC). Intracellular cAMP has been known to play an important role. Iseki et al. firstly discovered that photoactivated adenylyl cyclase (PAC) is a unique protein that can act both as a photoreceptor and as an effector to catalyse cAMP synthesis, in contrast with G-protein-coupled receptor systems, in which three different proteins act sequentially to modulate the level of cyclic nucleotide $[64,65]$. PAC was originally isolated from the flagellate Euglena gracilis. Following stimulation by blue light it causes a rapid increase in cAMP levels. Thus, using PAC allows manipulation of neurotransmitter release and behavior by directly affecting intracellular signaling $[66,67]$.

2.5. LiGluR. Volgraf et al. reported that an ionotropic glutamate receptor subtype 6 (iGluR6) was genetically and chemically engineered, rendering it light sensitive. Using structurebased design, they modified its ubiquitous clamshell-type ligand-binding domain to develop a light-activated channel, which was named LiGluR [68]. Their group also found that the LiGluR rapidly generated large currents (hundreds of $\mathrm{pA}$ ), yielding substantial (tens of $\mathrm{mV}$ ) depolarizations in hippocampal neurons. Millisecond flashes of light evoked action potentials or subthreshold voltage changes that mimic fast excitatory postsynaptic potentials. The ability to excite neurons with LiGluR compares favorably with the responses of ChR2 in that LiGluR currents were 5-fold larger, were stable during extended periods of illumination, and deactivated more quickly because deactivation was light-driven, thus enabling cells to be reliably fired at higher frequencies. In addition, LiGluR has the unique property that, once activated by a brief pulse of light, the channel will remain open for minutes in the dark, until a pulse of deactivating light closes it, thus enabling long depolarizations and trains of action potentials to be evoked with minimal light exposure [69].

2.6. HyLighter. Janovjak et al. also reported a potassiumselective ionotropic glutamate receptor that reversibly inhibits neuronal activity in response to light in dissociated neurons and brain slice and also reversibly suppresses behavior in zebrafish [70]. Chimeras were constructed in which the transmembrane helices and reentrant pore-loop of the $\mathrm{K}^{+}$-selective sGluR0 were transplanted into iGluR6, and the best of these was modified for light-gating and termed HyLighter. This hyperpolarizing light-gated channel is turned on by a brief light pulse at one wavelength and turned off by a pulse at a second wavelength. After optical activation, the photocurrent and optical silencing of activity persist in the dark for extended periods. The low light requirement and bistability of HyLighter represent advantages for the dissection of neural circuitry [70].

\section{Delivery of Light}

Electronic systems that integrate with the body provide powerful diagnostic and therapeutic capabilities for basic research and clinical medicine. Recent research establishes materials and mechanical constructs for electronic circuits, lightemitting diodes (LEDs), sensors, and other components. In optogenetics, fiber optic devices restrict opportunities for in vivo use and widespread biological application. As a solution, Bruchas and Rogers group developed mechanically compliant, ultrathin multifunctional optoelectronic systems that mount on releasable injection needles for insertion into the depths of soft tissue [71, 72]. These devices incorporate cellular-scale components ranging from independently addressable multicolored microscale. In addition, Tamura et al. reported a tungsten microelectrode-based optical probe, optrode, that encloses optical fibers within its insulation glass [73]. This smooth glass-coated optrode is a promising tool for chronic in vivo experiments with various research targets including deep brain structures in behaving monkeys.

Those types of custom device represent a significant step forward for the optogenetics community for in vivo applications, allowing multiple bright excitation sites along the length of a minimally invasive probe.

\section{Clinical Significance of Optogenetics}

The utility of optogenetics is not limited to experimental manipulations but also encompasses potentially significant therapeutic applications. Some of the most promising opportunities for clinical use of optogenetic methods have arisen, perhaps unsurprisingly, in the visual system, where ChR2's original function as a photosensitive pigment is most pertinent. When ChR2 was introduced into second-order neurons (ON bipolar cells) in the $r d 1$ mouse model of retinal degeneration, the resultant photosensitive cells were able to stimulate light-evoked responses in both retinal ganglion and visual cortex [74]. Likewise, introduction of ChR2 into retinal ganglion cells restored vision in genetically blind rats [75]. The results of these studies raise the possibility that this ChR2 could be used in gene therapy of certain forms of congenital or acquired blindness in humans [75].

In another field of medicine, ChR2 has been used to probe the roles of specific brain regions and activity patterns in governing seizures [76], revealing the potential significance 
of bidirectional networks in the hippocampus in determining the beginning and end of epileptic seizures. By facilitating development of methods to selectively block or activate relevant circuits, these findings may someday contribute to improved treatments for epilepsy.

Trauner and Isacoff group reported an approach whereby a genetically and chemically engineered LiGluR was expressed selectively in retinal ganglion cells (RGCs), the longest-surviving cells in retinal blinding diseases. When expressed in the RGCs of a well-established rat model of retinal degeneration, LiGluR restored light sensitivity to the RGCs, reinstated light responsiveness to the primary visual cortex, and restored both the pupillary reflex and a natural light-avoidance behavior [77].

\section{Conclusion}

Given the rate of progress over the last few years, it is reasonable to predict that the molecular techniques for optogenetics will continue to evolve rapidly and that the applications of these methods will continue to expand. Just as anatomical and genetic targeting of light-sensitive proteins had contributed tremendously to our knowledge of neural circuits, the growing tool set for subcellular targeting of optogenetic components should facilitate advances in our understanding of the importance of subcellular domains and intracellular compartments in the physiology and function of neurons and other cell types. Likewise, the control of proteinprotein interactions via light-activated dimerization domains will further enable controlled localization of proteins and production of signaling intermediates in specific subcellular regions.

From its origins in neuroscience, optogenetic technology had spread rapidly across disciplinary lines, and its vast range of potential applications has only begun to be sampled. As the technology grows and matures, optogenetics should continue to transform biology in the years to come.

\section{Conflict of Interests}

The authors declare that there is no conflict of interests regarding the publication of this paper.

\section{Acknowledgment}

This review article was supported by Japan Society for the Promotion of Science (JSPS) KAKENHI, Grant number 25713009.

\section{References}

[1] V. Plaiasu, "Update in genetics," Maedica (Buchar), vol. 6, no. 1, p. 70, 2011.

[2] G. Miesenböck, “The optogenetic catechism," Science, vol. 326, no. 5951, pp. 395-399, 2009.

[3] G. Miesenböck, "Optogenetic control of cells and circuits," Annual Review of Cell and Developmental Biology, vol. 27, pp. 731-758, 2011.
[4] T. Fehrentz, M. Schönberger, and D. Trauner, "Optochemical genetics," Angewandte Chemie, vol. 50, no. 51, pp. 12156-12182, 2011.

[5] M. Izquierdo-Serra, D. Trauner, A. Llobet, and P. Gorostiza, "Optical modulation of neurotransmission using calcium photocurrents through the ion channel LiGluR," Frontiers in Molecular Neuroscience, vol. 6, p. 3, 2013.

[6] K. Deisseroth, "Optogenetics," Nature Methods, vol. 8, no. 1, pp. 26-29, 2011.

[7] E. Pastrana, "Optogenetics: Controlling cell function with light," Nature Methods, vol. 8, no. 1, pp. 24-25, 2011.

[8] G. Kauwe and E. Y. Isacoff, "Rapid feedback regulation of synaptic efficacy during high-frequency activity at the Drosophila larval neuromuscular junction," Proceedings of the National Academy of Sciences of the United States of America, vol. 110, no. 22, pp. 9142-9147, 2013.

[9] C. Wyart, F. D. Bene, E. Warp et al., "Optogenetic dissection of a behavioural module in the vertebrate spinal cord," Nature, vol. 461, no. 7262, pp. 407-410, 2009.

[10] R. H. Kramer, A. Mourot, and H. Adesnik, "Optogenetic pharmacology for control of native neuronal signaling proteins," Nature Neuroscience, vol. 16, no. 7, pp. 816-823, 2013.

[11] G. Nagel, D. Ollig, M. Fuhrmann et al., "Channelrhodopsin-1: a light-gated proton channel in green algae," Science, vol. 296, no. 5577, pp. 2395-2398, 2002.

[12] G. Nagel, T. Szellas, W. Huhn et al., "Channelrhodopsin2, a directly light-gated cation-selective membrane channel," Proceedings of the National Academy of Sciences of the United States of America, vol. 100, no. 2, pp. 13940-13945, 2003.

[13] A. Matsuno-Yagi and Y. Mukohata, "ATP synthesis linked to light-dependent proton uptake in a red mutant strain of Halobacterium lacking bacteriorhodopsin," Archives of Biochemistry and Biophysics, vol. 199, no. 1, pp. 297-303, 1980.

[14] B. Schobert and J. K. Lanyi, "Halorhodopsin is a light-driven chloride pump," Journal of Biological Chemistry, vol. 257, no. 17, pp. 10306-10313, 1982.

[15] E. N. Spudich and J. L. Spudich, "Control of transmembrane ion flux to select halorhodopsin-deficient and other energytransduction mutants of Halobacterium halobium," Proceedings of the National Academy of Sciences of the United States of America, vol. 79, no. 14 I, pp. 4308-4312, 1982.

[16] E. S. Boyden, F. Zhang, E. Bamberg, G. Nagel, and K. Deisseroth, "Millisecond-timescale, genetically targeted optical control of neural activity," Nature Neuroscience, vol. 8, no. 9, pp. 1263-1268, 2005.

[17] K. Deisseroth, G. Feng, A. K. Majewska, G. Miesenböck, A. Ting, and M. J. Schnitzer, "Next-generation optical technologies for illuminating genetically targeted brain circuits," Journal of Neuroscience, vol. 26, no. 41, pp. 10380-10386, 2006.

[18] A. R. Adamantidis, F. Zhang, A. M. Aravanis, K. Deisseroth, and L. De Lecea, "Neural substrates of awakening probed with optogenetic control of hypocretin neurons," Nature, vol. 450, no. 7168, pp. 420-424, 2007.

[19] A. M. Aravanis, L. P. Wang, F. Zhang et al., "An optical neural interface: in vivo control of rodent motor cortex with integrated fiberoptic and optogenetic technology," Journal of neural engineering, vol. 4, no. 3, pp. S143-156, 2007.

[20] "Method of the Year 2010," Nature Methods, vol. 8, no. 1, 2010.

[21] A. M. Aravanis, L.-P. Wang, F. Zhang et al., "An optical neural interface: in vivo control of rodent motor cortex with integrated fiberoptic and optogenetic technology," Journal of neural engineering, vol. 4, pp. S143-S156, 2007. 
[22] B. R. Arenkiel, J. Peca, I. G. Davison et al., "In vivo light-induced activation of neural circuitry in transgenic mice expressing channelrhodopsin-2," Neuron, vol. 54, no. 2, pp. 205-218, 2007.

[23] E. S. Boyden, F. Zhang, E. Bamberg, G. Nagel, and K. Deisseroth, "Millisecond-timescale, genetically targeted optical control of neural activity," Nature Neuroscience, vol. 8, no. 9, pp. 1263-1268, 2005.

[24] X. Han and E. S. Boyden, "Multilpe-color optical activation, silencing, and desynchronization of neural activity, with singlespike temporal resolution," PLoS ONE, vol. 2, no. 3, article e299, 2007.

[25] T. Ishizuka, M. Kakuda, R. Araki, and H. Yawo, "Kinetic evaluation of photosensitivity in genetically engineered neurons expressing green algae light-gated channels," Neuroscience Research, vol. 54, no. 2, pp. 85-94, 2006.

[26] G. Nagel, M. Brauner, J. F. Liewald, N. Adeishvili, E. Bamberg, and A. Gottschalk, "Light activation of Channelrhodopsin2 in excitable cells of caenorhabditis elegans triggers rapid behavioral responses," Current Biology, vol. 15, no. 24, pp. 22792284, 2005.

[27] L. Petreanu, D. Huber, A. Sobczyk, and K. Svoboda, "Channelrhodopsin-2-assisted circuit mapping of long-range callosal projections," Nature Neuroscience, vol. 10, no. 5, pp. 663-668, 2007.

[28] C. Schroll, T. Riemensperger, D. Bucher et al., "Light-induced activation of distinct modulatory neurons triggers appetitive or aversive learning in Drosophila larvae," Current Biology, vol. 16, no. 17, pp. 1741-1747, 2006.

[29] J. B. Bryson, C. B. Machado, M. Crossley et al., "Optical control of muscle function by transplantation of stem cell-derived motor neurons in mice," Science, vol. 344, no. 6179, pp. 94-97, 2014.

[30] F. Zhang, L. P. Wang, M. Brauner et al., "Multimodal fast optical interrogation of neural circuitry," Nature, vol. 446, no. 7136, pp. 633-639, 2007.

[31] F. Zhang, L. P. Wang, E. S. Boyden, and K. Deisseroth, "Channelrhodopsin-2 and optical control of excitable cells," Nature Methods, vol. 3, no. 10, pp. 785-792, 2006.

[32] O. A. Sineshchekov, K. H. Jung, and J. L. Spudich, "Two rhodopsins mediate phototaxis to low- and high-intensity light in Chlamydomonas reinhardtii," Proceedings of the National Academy of Sciences of the United States of America, vol. 99, no. 13, pp. 8689-8694, 2002.

[33] X. Li, D. V. Gutierrez, M. G. Hanson et al., "Fast noninvasive activation and inhibition of neural and network activity by vertebrate rhodopsin and green algae channelrhodopsin," Proceedings of the National Academy of Sciences of the United States of America, vol. 102, no. 49, pp. 17816-17821, 2005.

[34] Y. P. Zhang and T. G. Oertner, "Optical induction of synaptic plasticity using a light-sensitive channel," Nature Methods, vol. 4, no. 2, pp. 139-141, 2007.

[35] B. K. Andrasfalvy, B. V. Zemelman, J. Tang, and A. Vaziri, "Twophoton single-cell optogenetic control of neuronal activity by sculpted light," Proceedings of the National Academy of Sciences of the United States of America, vol. 107, no. 26, pp. 11981-11986, 2010.

[36] G. Nagel, M. Brauner, J. F. Liewald, N. Adeishvili, E. Bamberg, and A. Gottschalk, "Light activation of Channelrhodopsin2 in excitable cells of caenorhabditis elegans triggers rapid behavioral responses," Current Biology, vol. 15, no. 24, pp. 22792284, 2005.
[37] B. R. Arenkiel, J. Peca, I. G. Davison et al., "In vivo light-induced activation of neural circuitry in transgenic mice expressing channelrhodopsin-2," Neuron, vol. 54, no. 2, pp. 205-218, 2007.

[38] D. Huber, L. Petreanu, N. Ghitani et al., "Sparse optical microstimulation in barrel cortex drives learned behaviour in freely moving mice," Nature, vol. 451, no. 7174, pp. 61-64, 2008.

[39] H. Wang, J. Peca, M. Matsuzaki et al., "High-speed mapping of synaptic connectivity using photostimulation in Channelrhodopsin-2 transgenic mice," Proceedings of the National Academy of Sciences of the United States of America, vol. 104, no. 19, pp. 8143-8148, 2007.

[40] H.-C. Tsai, F. Zhang, A. Adamantidis et al., "Phasic firing in dopaminergic neurons is sufficient for behavioral conditioning," Science, vol. 324, no. 5930, pp. 1080-1084, 2009.

[41] A. V. Kravitz, B. S. Freeze, P. R. L. Parker et al., "Regulation of parkinsonian motor behaviours by optogenetic control of basal ganglia circuitry," Nature, vol. 466, no. 7306, pp. 622-626, 2010.

[42] T. L. Lewis, T. Mao, K. Svoboda, and D. B. Arnold, "Myosindependent targeting of transmembrane proteins to neuronal dendrites," Nature Neuroscience, vol. 12, no. 5, pp. 568-576, 2009.

[43] A. V. Gourine, V. Kasymov, N. Marina et al., "Astrocytes control breathing through $\mathrm{pH}$-dependent release of ATP," Science, vol. 329, no. 5991, pp. 571-575, 2010.

[44] T. Bruegmann, D. Malan, M. Hesse et al., "Optogenetic control of heart muscle in vitro and in vivo," Nature Methods, vol. 7, no. 11, pp. 897-900, 2010.

[45] T. Asano, T. Ishizua, and H. Yawo, "Optically controlled contraction of photosensitive skeletal muscle cells," Biotechnology and Bioengineering, vol. 109, no. 1, pp. 199-204, 2012.

[46] J. P. Weick, M. A. Johnson, S. P. Skroch, J. C. Williams, K. Deisseroth, and S.-C. Zhang, "Functional control of transplantable human ESC-derived neurons via optogenetic targeting," Stem Cells, vol. 28, no. 11, pp. 2008-2016, 2010.

[47] A. Stroh, H. C. Tsai, L. P. Wang et al., "Tracking stem cell differentiation in the setting of automated optogenetic stimulation," Stem Cells, vol. 29, no. 1, pp. 78-88, 2011.

[48] R. D. Airan, K. R. Thompson, L. E. Fenno, H. Bernstein, and K. Deisseroth, "Temporally precise in vivo control of intracellular signalling," Nature, vol. 458, no. 7241, pp. 1025-1029, 2009.

[49] D. V. Gutierrez, M. D. Mark, O. Masseck et al., "Optogenetic control of motor coordination by Gi/o protein-coupled vertebrate rhodopsin in cerebellar purkinje cells," Journal of Biological Chemistry, vol. 286, no. 29, pp. 25848-25858, 2011.

[50] W. K. Karunarathne, L. Giri, V. Kalyanaraman, and N. Gautam, "Optically triggering spatiotemporally confined GPCR activity in a cell and programming neurite initiation and extension," Proceedings of the National Academy of Sciences of the United States of America, vol. 110, no. 17, pp. E1565-E1574, 2013.

[51] X. Wang, X. Chen, and Y. Yang, "Spatiotemporal control of gene expression by a light-switchable transgene system," Nature Methods, vol. 9, no. 3, pp. 266-269, 2012.

[52] X. Chen, X. Wang, Z. Du, Z. Ma, and Y. Yang, "Spatiotemporal control of gene expression in mammalian cells and in mice using the LightOn system," Current Protocols in Chemical Biology, vol. 5, no. 2, pp. 111-129, 2013.

[53] Z. Ma, Z. Du, X. Chen, X. Wang, and Y. Yang, "Fine tuning the LightOn light-switchable transgene expression system," Biochemical and Biophysical Research Communications, vol. 440, no. 3, pp. 419-423, 2013.

[54] Y. I. Wu, D. Frey, O. I. Lungu et al., "A genetically encoded photoactivatable Rac controls the motility of living cells," Nature, vol. 461, no. 7260, pp. 104-108, 2009. 
[55] M. A. Van Der Horst and K. J. Hellingwerf, "Photoreceptor proteins, "star actors of modern times": a review of the functional dynamics in the structure of representative members of six different photoreceptor families," Accounts of Chemical Research, vol. 37, no. 1, pp. 13-20, 2004.

[56] S. Masuda, "Light detection and signal transduction in the BLUF photoreceptors," Plant and Cell Physiology, vol. 54, no. 2, pp. 171-179, 2013.

[57] A. Möglich and K. Moffat, "Engineered photoreceptors as novel optogenetic tools," Photochemical and Photobiological Sciences, vol. 9, no. 10, pp. 1286-1300, 2010.

[58] S. Crosson, S. Rajagopal, and K. Moffat, "The LOV domain family: photoresponsive signaling modules coupled to diverse output domains," Biochemistry, vol. 42, no. 1, pp. 2-10, 2003.

[59] N. C. Rockwell, Y.-S. Su, and J. C. Lagarias, "Phytochrome structure and signaling mechanisms," Annual Review of Plant Biology, vol. 57, pp. 837-858, 2006.

[60] T. Kushibiki and K. Awazu, "Controlling osteogenesis and adipogenesis of mesenchymal stromal cells by regulating a circadian clock protein with laser irradiation," International Journal of Medical Sciences, vol. 5, no. 6, pp. 319-326, 2008.

[61] T. Kushibiki and K. Awazu, "Blue laser irradiation enhances extracellular calcification of primary mesenchymal stem cells," Photomedicine and Laser Surgery, vol. 27, no. 3, pp. 493-498, 2009.

[62] T. Kushibiki, T. Tajiri, Y. Ninomiya, and K. Awazu, "Chondrogenic mRNA expression in prechondrogenic cells after blue laser irradiation," Journal of Photochemistry and Photobiology B: Biology, vol. 98, no. 3, pp. 211-215, 2010.

[63] S. Konermann, M. D. Brigham, A. Trevino et al., "Optical control of mammalian endogenous transcription and epigenetic states," Nature, vol. 500, no. 7463, pp. 472-476, 2013.

[64] M. Iseki, S. Matsunaga, A. Murakami et al., "A blue-lightactivated adenylyl cyclase mediates photoavoidance in Euglena gracilis," Nature, vol. 415, no. 6875, pp. 1047-1051, 2002.

[65] S. Yoshikawa, T. Suzuki, M. Watanabe, and M. Iseki, "Kinetic analysis of the activation of photoactivated adenylyl cyclase (PAC), a blue-light receptor for photomovements of Euglena," Photochemical and Photobiological Sciences, vol. 4, no. 9, pp. 727-731, 2005.

[66] J. Looser, S. Schröder-Lang, P. Hegemann, and G. Nagel, "Mechanistic insights in light-induced cAMP production by photoactivated adenylyl cyclase alpha (PAC $\alpha$ )," Biological Chemistry, vol. 390, no. 11, pp. 1105-1111, 2009.

[67] S. Schröder-Lang, M. Schwärzel, R. Seifert et al., "Fast manipulation of cellular cAMP level by light in vivo," Nature Methods, vol. 4, no. 1, pp. 39-42, 2007.

[68] M. Volgraf, P. Gorostiza, R. Numano, R. H. Kramer, E. Y. Isacoff, and D. Trauner, "Allosteric control of an ionotropic glutamate receptor with an optical switch," Nature Chemical Biology, vol. 2, no. 1, pp. 47-52, 2006.

[69] S. Szobota, P. Gorostiza, F. del Bene et al., "control of neuronal activity with a light-gated glutamate receptor," Neuron, vol. 54, no. 4, pp. 535-545, 2007.

[70] H. Janovjak, S. Szobota, C. Wyart, D. Trauner, and E. Y. Isacoff, "A light-gated, potassium-selective glutamate receptor for the optical inhibition of neuronal firing," Nature Neuroscience, vol. 13, no. 8, pp. 1027-1032, 2010.

[71] T. i. Kim, J. G. McCall, Y. H. Jung et al., "Injectable, cellular-scale optoelectronics with applications for wireless optogenetics," Science, vol. 340, no. 6129, pp. 211-216, 2013.
[72] J. G. McCall, T. I. Kim, G. Shin et al., "Fabrication and application of flexible, multimodal light-emitting devices for wireless optogenetics," Nature Protocols, vol. 8, no. 12, pp. 2413-2428, 2013.

[73] K. Tamura, Y. Ohashi, T. Tsubota et al., "A glass-coated tungsten microelectrode enclosing optical fibers for optogenetic exploration in primate deep brain structures," Journal of Neuroscience Methods, vol. 211, no. 1, pp. 49-57, 2012.

[74] P. S. Lagali, D. Balya, G. B. Awatramani et al., "Light-activated channels targeted to ON bipolar cells restore visual function in retinal degeneration," Nature Neuroscience, vol. 11, no. 6, pp. 667-675, 2008.

[75] H. Tomita, E. Sugano, H. Isago, and M. Tamai, "Channelrhodopsins provide a breakthrough insight into strategies for curing blindness," Journal of Genetics, vol. 88, no. 4, pp. 409$415,2009$.

[76] S. Osawa, M. Iwasaki, R. Hosaka et al., "Optogenetically induced seizure and the longitudinal hippocampal network dynamics," PLoS ONE, vol. 8, no. 4, Article ID e60928, 2013.

[77] N. Caporale, K. D. Kolstad, T. Lee et al., "LiGluR restores visual responses in rodent models of inherited blindness," Molecular Therapy, vol. 19, no. 7, pp. 1212-1219, 2011. 

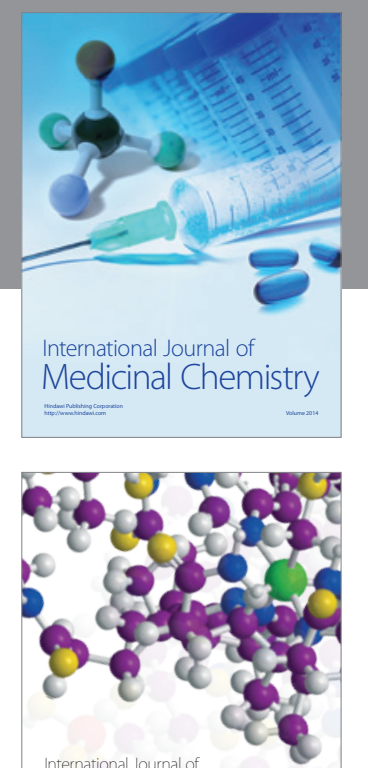

\section{Carbohydrate} Chemistry

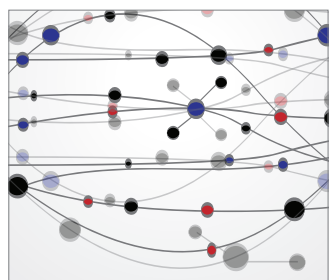

The Scientific World Journal
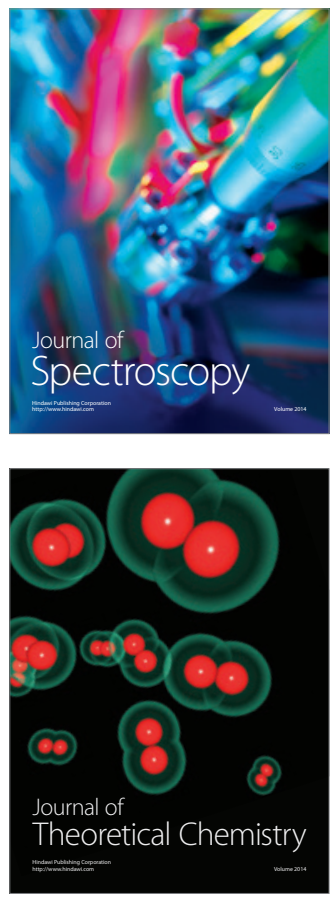
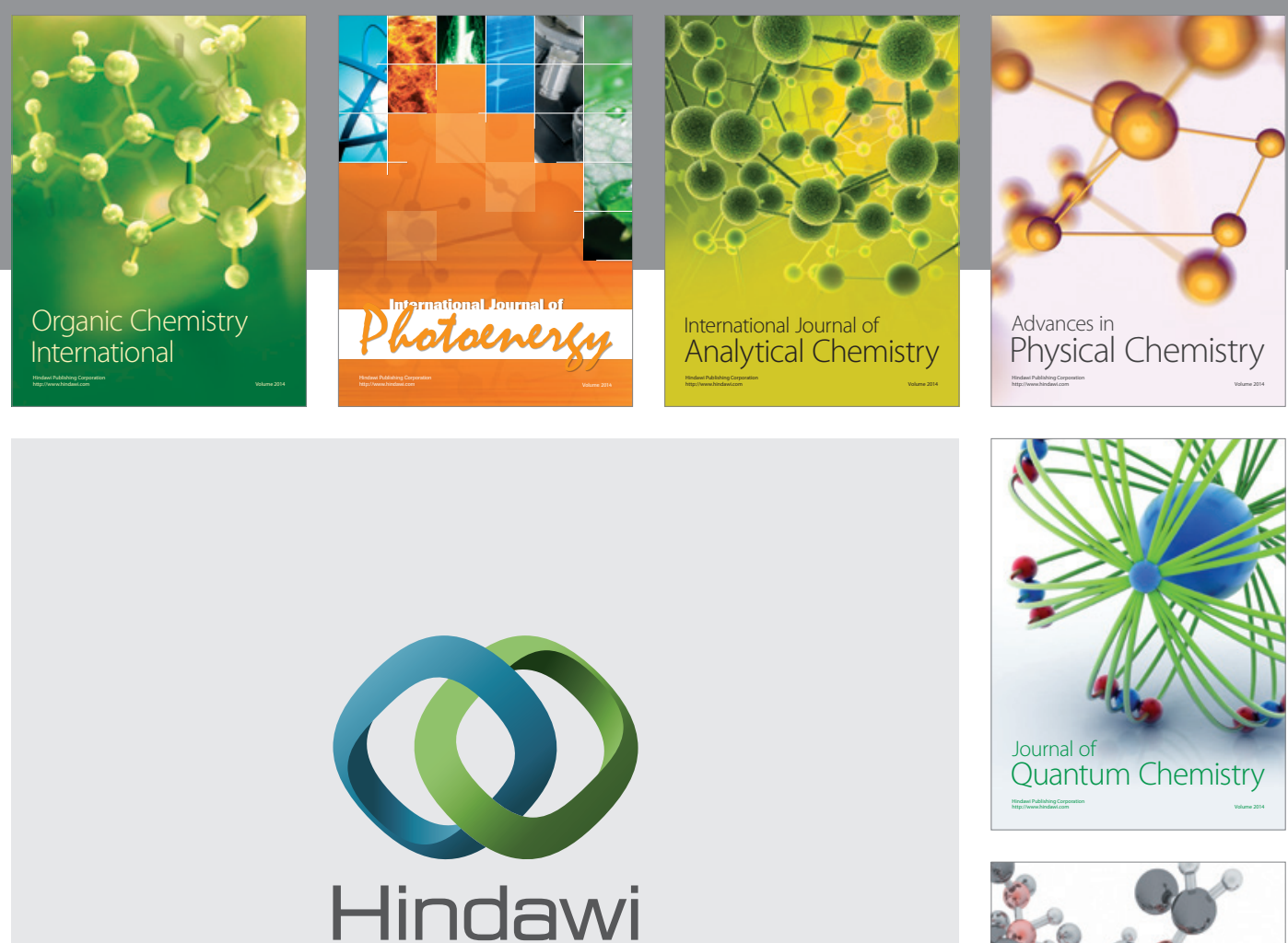

Submit your manuscripts at

http://www.hindawi.com

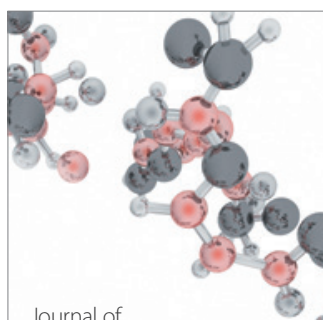

Analytical Methods

in Chemistry

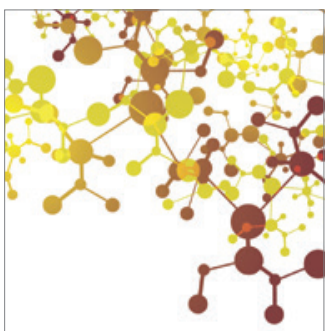

Journal of

Applied Chemistry

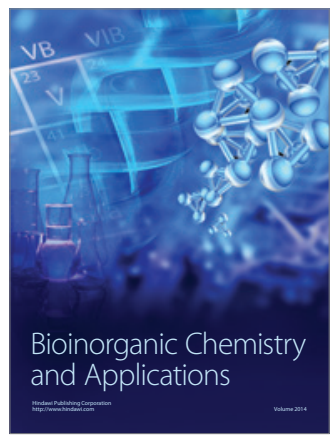

Inorganic Chemistry
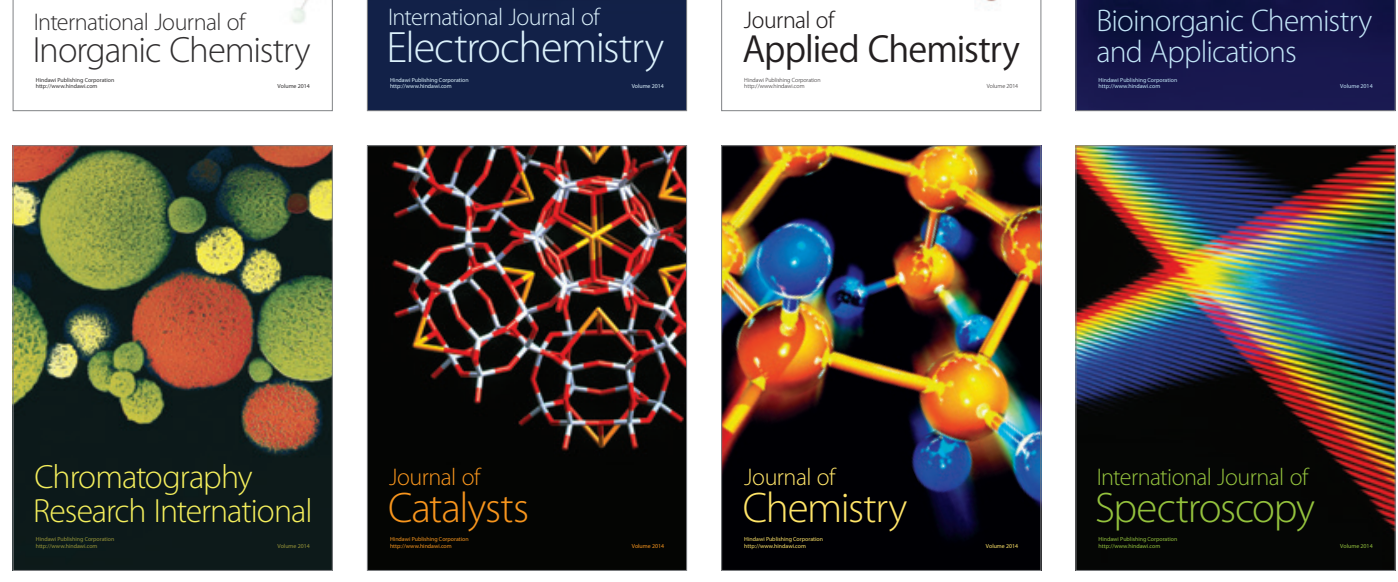